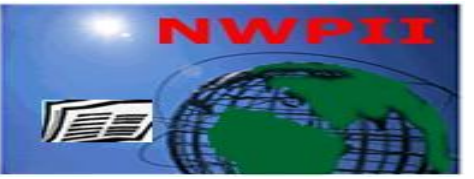

American Journal of Biomedical Sciences

ISSN: 1937-9080

nwpii.com/ajbms

\title{
Comparison of Broth Dilution and Disc Diffusion Method for the Antifungal Susceptibility Testing of Aspergillus flavus
}

\author{
Ravi Kumar ${ }^{1}$, Sandeep Kumar Shrivastava ${ }^{2,3}$, Arunaaloke Chakraborti ${ }^{4}$ \\ ${ }^{1}$ Department of Microbiology, BMS Block, Panjab University, Chandigarh, India-160014 \\ ${ }^{2}$ Amity Institute of Biotechnology, AMITY University Rajasthan, Jaipur, India \\ ${ }^{3}$ Center for Infection and Immunity of Lille, INSERM U1019 - CNRS UMR 8204, Institute Pasteur de Lille, 59019 \\ Lille Cedex, France \\ ${ }^{4}$ Division of Medical Mycology, Department of Medical Microbiology, Post Graduate Institute of Medical Education \\ and Research, Chandigarh, India-160014 \\ *Corresponding Author: \\ Ravi Kumar \\ Department of Microbiology \\ BMS Block, Sector 14 \\ Panjab University \\ Chandigarh-160014, India \\ Phone: 91-172-2534142 \\ Fax: 91-172-2547170 \\ Email: ravigupta2007@ hotmail.com
}

Received: 1 September 2009; | Revised: 3 March 2010; | Accepted: 20 March 2010

\begin{abstract}
With the discovery of newer antifungal agents, emergence of resistance among Aspergillus, is a major problem. In the present study, 25 different clinical isolates of Aspergillus flavus were screened for their susceptibility against five common antifungal agents by broth dilution and disc diffusion method with the aim to evaluate both the methods for their suitability to adapt in clinical laboratory. All the isolates showed susceptibility to five antifungals by broth dilution after $24 \mathrm{hrs}$ however all isolates showed resistance for 5FC, fluconazole and ketoconazole after $48 \mathrm{hrs}$. By disc diffusion method, all isolates were sensitive for all antifungals except fluconazole which showed resistance even after $24 \mathrm{hrs}$. Amphotericin B and itraconazole were in good agreement between both the methods where $100 \%$ isolates were sensitive at both time period by broth dilution and $84 \%$ to $100 \%$ by disc diffusion method. Both the methods are in good agreement and since disc diffusion method is easy to perform, cheap, rapid and same can be adapted easily for the routine susceptibility testing of clinical fungal isolates in medical microbiology laboratory.
\end{abstract}

Keywords: Aspergillosis; Aspergillus flavus; antifungals; susceptibility testing; disc diffusion; broth dilution; NCCLS.

\section{Introduction}

Am. J. Biomed. Sci. 2010, 2(3), 202-208; doi: 10.5099/aj100300202 @ 2010 by NWPII. All rights reserved. 
Invasive aspergillosis has emerged worldwide as an important cause of infection among immunocompromised patients (Bodey et al., 1992; Groll et al., 1996; Denning et al., 1996). Aspergillosis is a serious opportunistic mycotic infection caused by the genus Aspergillus majorly by Aspergillus fumigatus followed by Aspergillus flavus and Aspergillus niger (Rippon, 1982). The spectrum of aspergillosis includes ABPA, colonization of air sacs, invasive aspergillosis, pulmonary, aspergillosis in solid organs transplantation and invasive aspergillosis in AIDS patients. Treating the patients with aspergillosis is difficult especially in immunocompromised and organ transplantation patients and becomes cause of mortality rate of approximately $85 \%$ if not treated (Rippon, 1988; Denning et al., 1996, 2002; Maertens et al., 2004).

Diagnosis and treatment of invasive aspergillosis is one of the unmet needs in medicine today (Lin et al., 2001; Paterson, 2001). Mortality rate can be reduced if the disease is diagnosed and treated at early stage. Various newer broad spectrum antifungals like new triazoles, posaconazole, ravuconazole, and voriconazole were discovered in recent time but simultaneous emergence of resistance becomes cause of concern (NCCLS 2002; Meletiadis et al., 2002). To overcome this, susceptibility testing is one approach. Although different methods like broth dilution and agar dilution were developed in recent time for the antifungal susceptibility testing but due to cumbersome procedure, were not easy to adapt in the routine practice specially in case of filamentous fungi. Very few reports are available regarding antifungals susceptibility testing of filamentous fungi. Brandsberg et al., (1972) used conidial spore suspension of Sporothrix schenckii and Aspergillus fumigatus to find out the MIC against amphotericin B. Bez (1985) compared hyphal and conidial inoculums for the susceptibility testing of amphotericin B and found that use of conidial suspension was more appropriate than hyphal inoculum. Szekely et al., (1999) compared the E-test and microbroth dilution method for susceptibility testing of filamentous fungi against amphotericin-B and itraconazole and found that $\mathrm{E}$ test was more advantageous than broth dilution. Predicting antifungal drug resistance of an organism to available drugs is the goal of antifungal susceptibility testing, which, in turn, might aid in timely and successful intervention of these lifethreatening infections (Rex and Pfaller, 2002). Number of antifungal susceptibility approaches has been tried so far but due to inter and intra laboratory dis-agreement and lack of reproducibility, were not adapted in routine practice. In the present study, broth dilution and disc diffusion methods were evaluated for antifungal susceptibility testing of filamentous fungi, Aspergillus flavus against five common antifungal agents for their suitability to adapt in routine laboratory practices.

\section{Material and methods}

Clinical Isolates: 25 different Aspergillus flavus strains isolated from various clinical samples (Table 1) and maintained in Reference Laboratory for Collection of Medically Important Fungi, Division of Medical Mycology, Department of Microbiology, Post Graduate Institute of Medical Education and Research (PGIMER), Chandigarh, India were used for antifungal susceptibility testing. All the clinical isolates were identified as Aspergillus flavus by conventional method (Kwonchung., 1992). Strains were maintained by subculture method on potato malt agar and stored at $4^{0} \mathrm{C}$.

Antifungals: Antifungals in purified form obtained from respective manufacturers as Fluconazole (Pfizer Pharmaceuticals, India), 5Flucytosine (Sigma Chemicals, USA), Amphotericin B (Himedia Lab. Pvt. Ltd, Mumbai), Ketoconazole (Torrent Laboratories, India) and Itraconazole (Janssen Pharmaceuticals, Belgium) were used for the antifungal susceptibility testing.

Antifungal susceptibility testing: Susceptibility testing was done by employing broth dilution and disc diffusion method as described below.

Broth Dilution Method: Broth dilution method was done following the standard method of NCCLS M38A.

Preparation of Inoculum: For broth dilution method, spores were collected from 7 days old 
culture of Aspergillus flavus with the help of sterile swab dipped in normal saline $(0.85 \% \mathrm{w} / \mathrm{v})$ containing Tween-80 $(0.1 \% \quad \mathrm{v} / \mathrm{v}, \quad$ Sigma Chemicals, USA). Spore count of $1 \times 10^{6}$ spores $/ \mathrm{ml}$ was adjusted by haemocytometer count. Inoculum was further diluted 1:100 followed by $1: 20$ in RPMI 1640 to finally yield $0.5-2.5 \times 10^{3}$ spores $/ \mathrm{ml}$.
Antifungal Stock Preparation: Stock solution of antifungals amphotericin B, ketoconazole and intraconazole was prepared in dimethyl sulphoxide (DMSO, Sigma chemicals, USA) while fluconazole and 5 flucytosine were dissolved in sterile de-ionized water.

Table 1: Table showing number of clinical isolates of Aspergillus flavus isolated from different infection sites and samples for the drug susceptibility testing.

\begin{tabular}{|l|l|l|}
\hline Sr. No. & Sites/samples of Aspergillus flavus isolation & Total No. of isolates \\
\hline 1. & Paranasal sinus mycoses (Tissue) & 13 \\
\hline 2. & Broncho alveolar lavage & 6 \\
\hline 3. & Sputum & 2 \\
\hline 4. & Cerebro spinal fluid & 1 \\
\hline 5. & Skin biopsy & 1 \\
\hline 6. & Fine needle aspiration material & 1 \\
\hline 7. & Chest tube & 1 \\
\hline
\end{tabular}

Antifungal susceptibility testing: $100 \mu \mathrm{l}$ of different antifungals dilutions (Water soluble at $640 \mu \mathrm{g} / \mathrm{ml}$ to $0.3125 \mu \mathrm{g} / \mathrm{ml}$ and water insoluble at $160 \mu \mathrm{g} / \mathrm{ml}$ to $0.3125 \mu \mathrm{g} / \mathrm{ml}$ ) were placed in different tubes. After this, $0.9 \mathrm{ml}$ of adjusted spore inoculums was added to these tubes. This resulted in further 1:10 drug dilution with final concentration from $64 \mu \mathrm{g}$ to $0.03125 \mu \mathrm{g} / \mathrm{ml}$ and $16 \mu \mathrm{g}$ to $0.03125 \mu \mathrm{g} / \mathrm{ml}$ respectively. One tube without antifungal agent with spore inoculum was used as positive control. All the tubes were incubated at $37^{0} \mathrm{C}$ for 24 to $48 \mathrm{hrs}$.

Disc Diffusion Method: Disc diffusion test was done according to the method of NCCLS M27A2 (2002). Yeast nitrogen base glucose (YNBG) with $1.5 \% \mathrm{~L}$-asparagine for azoles and without asparagines for amphotericin B and 5 flucytosine was prepared and filter sterilized. Antifungal discs containing $10 \mu \mathrm{l}$ of antifungal agents $(1 \mathrm{mg} / \mathrm{ml})$ were prepared and dried for use. For susceptibility testing, $2 \mathrm{ml}$ of YNBG was thoroughly mixed with $18 \mathrm{ml}$ of molten agar $(2 \%$ ultra purified agar, Diffco Labs, USA) and poured in $100 \mathrm{~mm}$ sterile petri plates. Plates were dried prior to use. Plates were inoculated with adjusted spore suspension $\left(1 \times 10^{6} / \mathrm{ml}\right)$ with sterile swab. One disc of each antifungal was placed in each plate and was incubated at $37^{\circ} \mathrm{C}$ for 24 to $48 \mathrm{hrs}$.

\section{Results and discussion}

Fungal infections are becoming an increasing cause of morbidity and mortality especially among immunocompromised patients. With the increased incidence of systemic fungal infections and the growing number of antifungal agents, laboratory methods to guide and select antifungal therapy 
have gained greater attention. However, determining antifungal susceptibilities of filamentous fungi is fraught with difficulties associated with slow growth of filamentous forms and subjectivity of interpreting visual endpoints.

In the present study, antifungal susceptibility testing of 25 clinical isolates was observed against five common antifungal agents. MIC of all the isolates against antifungals was recorded after 24 and 48 hrs. For amphotericin B and itraconazole, MIC was read as the lowest drug concentration which didn't showed any visual growth $(100 \%$ inhibition). For flucytosine, fluconazole and ketoconazole, end point corresponding to approximately $50 \%$ or more reduction in growth as compared to control was used (NCCLS M38A). In disc diffusion method, presence of inhibition zone was considered as sensitive while absence of inhibition zone was recorded as resistant. After 24 $\mathrm{hr}, 100 \%$ strains were sensitive to all the antifungals (5FC, fluconazole, ketoconazole, amphotericin B and itraconazole) by broth dilution method. After 48 hrs, no strain was sensitive to 5 flucytosine and only $4 \%$ strains were sensitive to fluconazole. Only $8 \%$ strains were sensitive to ketoconazole after $48 \mathrm{hrs}$ of incubation. In case of amphotericin B and itraconazole, $100 \%$ strains remained sensitive after $48 \mathrm{hrs}$ with slight increase in MIC (Figure 1). It was also observed that MIC increased with the increase in incubation period (Table 2). Susceptibility testing by disc diffusion method showed that no strain was sensitive to fluconazole at any time interval while 5 flucytosine showed sensitivity to $100 \%$ and $48 \%$ stains after 24 and $48 \mathrm{hrs}$ of incubation respectively. Ketoconazole also showed $100 \%$ and $60 \%$ sensitivity at 24 and $48 \mathrm{hr}$ of incubation. Against itraconazole, $100 \%$ strains were sensitive after $24 \mathrm{hr}$ while $84 \%$ were sensitive after $48 \mathrm{hr}$. Amphotericin B showed 96\% and $88 \%$ sensitivity after 24 and $48 \mathrm{hr}$ respectively (Figure 1).

Table 2: Table showing the breakpoint concentrations (NCCLS M38A) and MIC's observed by broth dilution method after 24 and $48 \mathrm{hrs}$ of incubation against clinical isolates of Aspergillus flavus against five antifungal agents.

\begin{tabular}{|l|l|l|l|}
\hline \multirow{2}{*}{ Antifungal } & Breakpoint & MIC's observed \\
\cline { 3 - 4 } & \multirow{2}{*}{ concentration } & After $24 \mathrm{hrs}(\mu \mathrm{g} / \mathrm{ml})$ & After $48 \mathrm{hrs}(\mu \mathrm{g} / \mathrm{ml})$ \\
& $(\mu \mathrm{g} / \mathrm{ml})$ & & \\
\hline Amphotericin B & $0.5-2.0$ & $0.0625-0.125$ & $0.125-0.250$ \\
\hline 5 Flucytosine & $\square 64$ & $8-32$ & $\square 64$ \\
\hline Fluconazole & $\square 64$ & $4-32$ & $32-\square 64$ \\
\hline Ketoconazole & 0.0313 to 16 & $0.5-1$ & $1-64$ \\
\hline Itraconazole & 0.0313 to 16 & $0.0313-0.0625$ & $0.0625-0.125$ \\
\hline
\end{tabular}

In the present study, MIC was observed after 24 and $48 \mathrm{hr}$ and it was observed that interpretation of results were better at $24 \mathrm{hr}$ than $48 \mathrm{hr}$. It was observed that susceptibility of 5-FC, fluconazole and ketoconazole was affected more than that of amphotericin B and intraconazole. Increase in MIC may indicate towards the emergence of resistance. It has been indicated that antimicrobial resistance may also be indicative of therapeutic failure (Ashok R, 1999). Various 
reports have shown the emergence of resistance among filamentous fungi for different antifungals. It has been observed that 5 flucytosine and fluconazole are ineffective for the treatment of aspergillosis (Ryley JF et al., 1990) but in the present study, all the isolates were observed to be sensitive to these two antifungals after 24 hours of incubation. Ketoconazole has also not been considered as good drug of choice for the treatment of aspergillosis but it has also been observed that results of in vitro susceptibility may be of less relevance and misleading in case of ketoconazole. Since amphotericien B and itraconazole showed less resistance than other three antifungals, it may be concluded that both these drugs might have a definite chemotherapeutic outcome in aspergillosis. Several reports have also described in vitro tolerance/resistance of Aspergillus spp. which correlates with therapeutic failure (Florl et al., 1998; Sutton et al., 1999; Mosqurel et al., 2001). Ingroff et al., (2001) have observed resistance to amphotericin B particularly among isolates of Aspergillus terreus and other filamentous fungi, such as Fusarium spp. and Scedosporium spp. Very little data are available regarding correlation between MIC and outcome of treatment with amphotericin B for the filamentous fungi. MICs above $2 \mu \mathrm{g} / \mathrm{ml}$ have been associated with treatment failures while MICs below $2 \mu \mathrm{g} / \mathrm{ml}$ have been showed to be effective in clinical cure for invasive aspergillosis (Lass-Florl et al., 1998). Similarly, trailing end points with itraconazole against Aspergillus spp. are not usually encountered but it may also reflect drug resistance relevant clinically as intraconazole resistance has also been demonstrated for A. fumigatus (Espinel-Ingroff A et al., 2001; Denning DW et al., 1997).

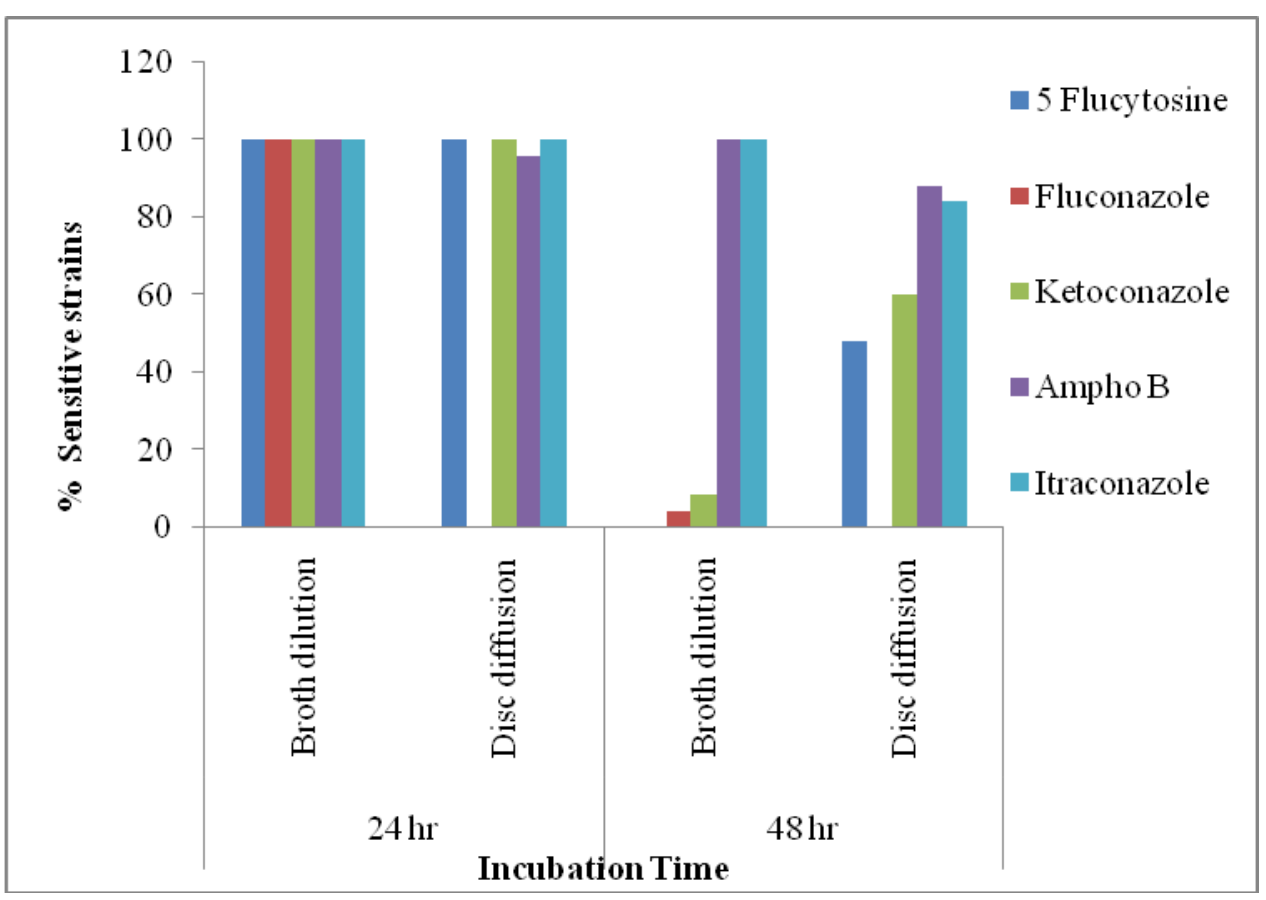

Figure 1. Figure showing the distribution of percentage strains of Aspergillus flavus sensitive to five antifungal agents after 24 and 48 hours of incubation by broth dilution and disc diffusion method.

The purpose of the present study was to compare the broth dilution and disc diffusion method efficacy for the susceptibility testing so as to adapt in routine. Although broth dilution method is the recommended standard method by NCCLS but this method is associated with some technical drawbacks such as cumbersome procedure, time consuming, technically demanding with poor end point precision particularly when fungistatic agents such as azole and 5-FC are tested. It has also been associated with the difficulty of preparation of standard 
hyphal inoculum. While on the other hand disc diffusion method is easy to perform, rapid and cost effective. It has been demonstrated that disc diffusion method is more useful as compared to other methods for antifungal drug susceptibility testing. Soraino et al., (2004) showed the advantage of disc diffusion method over other methods such as broth dilution or $E$ test for voriconazole susceptibility testing of Aspergillus. Yamaguchi et al., (2002) demonstrated usefulness of colorimeteric assay for the susceptibility testing of voriconazole, 5FC and itraconazole against Aspergillus over conventional methods. In another study carried out by Theres et al., (2006), it was observed that agar dilution method is more easy and cost effective for the susceptibility testing of filamentous fungi against amphotericin B, fluconazole and ketoconazole over broth dilution method. Very few reports are available on comparative analysis of disc diffusion and broth dilution method for susceptibility testing of filamentous fungi. In the present study, it was observed that after 24 hrs of incubation, both methods were in good agreement to each other except for fluconazole which is usually not a drug of choice for aspergillosis. Since disc diffusion method is easy and rapid to perform, it can be adapted easily for the routine antifungal susceptibility testing of filamentous fungi in clinical laboratory. Prediction of rapid antifungal susceptibility may improve and increase the speed of therapeutic outcome of infections caused by aspergillus.

\section{References}

1. Bodey, G.P.; Bueltmann, B.; Duguid, W. Fungal infections in cancer patients: an international autopsy study, Eur J Clin Microbiol Infect Dis, 1992, 11: 99-109.

2. Groll, A.; Shah, P.; Menzel, C. Trends in the postmortem epidemiology of invasive fungal infections at a university hospital, J Infect, 1996, 33: 23-32.

3. Denning, D.W. Therapeutic outcome in invasive aspergillosis, Clin Infect Dis, 1996, 23: 608-615.
4. Denning, D.W.; Ribaud, P.; Milpied, N. Efficacy and safety of voriconazole in the treatment of acute invasive aspergillosis, Clin Infect Dis, 2002, 34: 563-571.

5. Maertens, J.; Raad, I.; Petrikkos, G.; Boogaerts, M. Efficacy and safety of caspofungin for treatment of invasive aspergillosis in patients refractory to or intolerant to conventional antifungal therapy, Clin Infect Dis, 2004, 39: 1563-1571.

6. Rippon, J.W. Medical Mycology, 1988, $3^{\text {rd }} \mathrm{Ed}$. The W B Saunders Co, Philadelphia, p 618650.

7. Lin, S.J.; Schranz, J.; Teutsch, S.M. Aspergillosis case-fatality rate: systematic review of the literature, Clin Infect Dis, 2001, 32:358-366.

8. Patterson, T. F. Invasive mycoses: management and unmet medical needs: editorial review, Curr Opin Infect Dis, 2001, 14:669-671.

9. National Committee for Clinical Laboratory Standards. Reference method for broth dilution antifungal susceptibility testing of filamentous fungi: Approved standard M38-A, 2002, National Committee for Clinical Laboratory Standards, Wayne, Pa.

10. NCCLS. Reference Method for Broth Dilution Antifungal Susceptibility Testing of Yeasts; Approved Standard M27-A2, 940 West Valley Road, Suite 1400, Wayne, Pennsylvania 19087-1898, USA 2002.

11. Meletiadis, J.J.S.; Mouton, J. F. G. M.; Meis, B. A.; Bouman, P. E.; Verweij, and the EUROFUNG Network. Comparison of the Etest and the Sensititre colorimetric methods with the NCCLS proposed standard for antifungal susceptibility testing of Aspergillus species, J Clin Microbiol, 2002, 40:28762885.

12. Espinel-Ingroff, A.; and Kerkering, T. M. Spectrophotometric method of inoculum preparation for the in vitro susceptibility testing of filamentous fungi, J Clin Microbiol, 1991, 29:393-394.

13. Brandsberg, J.W. and French, M.E. In vitro susceptibility of isolates of Aspergillus fumigatus and Sporotherix schenkii to 
amphotericin B, Antimicrob Agents Chemother, 1972, 2(5): 402-404.

14. Bez, J.A.K. Standardization of a hyphal inoculum of Aspergilli for amphotericin B susceptibility testing, 1985, 21(4) 509-512.

15. Szekely, A.; Elizabeth, M.; Johnson, David, W.Warnock. Comparison of E-Test and Broth Microdilution Methods for Antifungal Drug Susceptibility Testing of Molds, JCM, 1999, p. 1480-1483.

16. Rex, J. H. and Pfaller, M.A. Has antifungal susceptibility testing come of age?, Clin Infect Dis, 2002, 35:982-989.

17. Kwon-Chung, K.J.; Bennet, J.E. Medical Mycology, Aspergillosis, Lea and Febiger, 1992, 201-247.

18. Ashok, R. Antifungal susceptibility testing, IJMR, 1999, 17(3);125-128.

19. Ryley, J.F. Screening and evaluation in vivo chemotherapy of fungal diseases in Hand book of experimental pharmacology, 1990, 96:129145.

20. Lass-Florl, C. G.; Kofler, G.; Kropshofer, J.; Hermans, A.; Kreczy, M. P.; Dierich, Niederwieser, D. In-vitro testing of susceptibility to amphotericin B is a reliable predictor of clinical outcome in invasive aspergillosis, J Antimicrob Chemother, 1998, 42:497-502.

21. Sutton, D. A.; Sanche, S.E.; Revankar, S.G.; Fothergill, A.W.; Rinaldi, M.G. In vitro amphotericin B resistance in clinical isolates of Aspergillus terreus, with a head-to-head comparison to voriconazole, J Clin Microbiol, 1999, 37:2343-2345.

22. Mosqueral, J.; Warn, P.A.; Morrissey, J.; Moore, C.B.; Gil-Lamaignere, C.; Denning, D.W. Susceptibility testing of Aspergillus flavus: inoculum dependence with itraconazole and lack of correlation between susceptibility to amphotericin B in vitro and outcome in vivo, Antimicrob Agents Chemother, 2001, 45:1456-1462.

23. Espinel-Ingroff, A. In vitro fungicidal activities of voriconazole, itraconazole, and amphotericin B against opportunistic moniliaceous and dematiaceous fungi, J Clin Microbiol, 2001, 39:954-958.

24. Denning, D. W.; Radford, S.A.; Oakley, K.L.; Hall, L.; Johnson, E.M.; Warnock. D.W. Correlation between in-vitro susceptibility testing to itraconazole and in-vivo outcome of Aspergillus fumigatus infection, $\mathrm{J}$ Antimicrob Chemother, 1997, 40:401-414.

25. Serraino, M. C.; Ramirez, I.; Morilla, M.; Valverde, D.; Chavez, M.; Espinel-Ingroff, A.; Claro, R.; Fernandez, A.; Almeida, C.; MartinMazuelos, E. A comparative study of the disc diffusion method with the broth microdilution and Etest methods for voriconazole susceptibility testing of Aspergillus spp, J Antimicrob Chemo, 2004, 53(5): 739-742.

26. Yamaguchi, H.; Uchida, K.; Nagino, K.; Matsunaga, T. Usefulness of a colorimetric method for testing antifungal drug susceptibilities of Aspergillus species to voriconazole, J Infect Chemother, 2002, 8:374-377.

27. Therese, K.L.; Bhagyalakshmi, R.; Madhvan, H.N.; Deepa, P. In vitro antifungal susceptibility testing by agar dilution method to determine the MIC of amphotericin B, fluconazole and ketoconazole against ocular fungal isolates, IJMM, 2006, 24(4) 273-9. 